Case Study

\title{
Physical therapy for multiple myeloma patients with severely hindered daily living activities due to bone lesions: a report of two cases
}

\author{
RYOHEI Jinbo, RPT ${ }^{1)^{*}}$, TAKAAKI FuJita, OTR, PhD ${ }^{2}$, RYUICHI KASAHARA, RPT, MS ${ }^{1)}$, \\ Shinichiro Morishita, RPT, PhD ${ }^{3)}$, Yuichi Yamamoto, RPT ${ }^{1)}$, KazUmi Jinbo, RPT ${ }^{1)}$, \\ Aya Takano, RPT ${ }^{1)}$, Junko Kubota, RPT ${ }^{1)}$, Shoko Takahashi, RPT ${ }^{1)}$, \\ Yutaka Shiga, MD, PhD ${ }^{4}$, Hideo Kimura, MD, PhD ${ }^{4)}$, Miki Furukawa, MD, $\mathrm{PhD}^{4}$, \\ TATSUYUKi KAI, MD, $\mathrm{PhD}^{4)}$ \\ 1) Department of Rehabilitation, Kita-Fukushima Medical Center: 23-1 Aza-higashi, Hakozaki, \\ Date-shi, Fukushima 960-0502, Japan \\ 2) Department of Occupational Therapy, School of Health Sciences, Fukushima Medical University, \\ Japan \\ 3) Department of Physical Therapy, School of Health Sciences, Fukushima Medical University, Japan \\ 4) Department of Hematology, Kita-Fukushima Medical Center, Japan
}

\begin{abstract}
Purpose] Physical therapy for patients with multiple myeloma requires appropriate exercise intensity and risk management due to osteolytic lesions. However, the optimal strategy for setting exercise intensity remains unclear. We report cases in which physical therapy was performed using the Borg scale and the Common Terminology Criteria for Adverse Events v4.0 as indicators of improvement in the performance of activities of daily living without causing adverse events. [Participants and Methods] Two patients with multiple myeloma, whose performance status was 4, underwent resistance training of the upper and lower limbs and activities of daily living practice in stages according to their functional status. Each exercise was performed for 20 to 40 minutes twice a day for 6 days a week. The exercise intensity was set to 13 on the Borg scale as a guide, and the allowable bone pain was up to Grade 1 according to Common Terminology Criteria for Adverse Events v4.0. [Results] No adverse events occurred in either patient, and the performance status improved to 1 or 2 . Subsequently, autologous peripheral hematopoietic stem cell transplantation was performed. [Conclusion] Physical therapy with exercise intensity set to 13 on the Borg scale and Grade 1 per Common Terminology Criteria for Adverse Events v4.0 may safely improve the performance of activities of daily living of patients with multiple myeloma.

Key words: Multiple myeloma, Borg scale, Physical therapy intervention
\end{abstract}

(This article was submitted May 16, 2021, and was accepted Jul. 1, 2021)

\section{INTRODUCTION}

Multiple myeloma (MM) is a hematological malignancy in which plasma cells become tumorous during the final stage of B-lymphocyte differentiation. It has a poor prognosis, characterized by renal damage, anemia, immunodeficiency, and osteolytic lesions. Approximately $76 \%$ patients have osteolytic lesions and about $46 \%$ have pathologic fractures of the spine $^{1)}$. Pathologic fractures or paralysis caused by bone lesions can significantly impact activities of daily living (ADLs) and quality of life ${ }^{2}$.

Very few reports have shown the efficacy and safety of physical therapy (PT) for MM patients. PT for patients with MM

*Corresponding author. Ryohei Jinbo (E-mail: reha-jinbo@jinsenkai.or.jp)

(C2021 The Society of Physical Therapy Science. Published by IPEC Inc.

(c) (i) $(-)$ This is an open-access article distributed under the terms of the Creative Commons Attribution Non-Commercial No DerivaCC BY NC ND tives (by-nc-nd) License. (CC-BY-NC-ND 4.0: https://creativecommons.org/licenses/by-nc-nd/4.0/) 
has some risk for pain, pathologic fractures, and paralysis, making careful planning vital. Regarding the safety, there are reports on the safety of PT in patients with bone metastases ${ }^{3-5)}$, while very few practical or case reports on PT or proper exercise intensity for patients with MM are present in the existing literature. Therefore, the optimal strategy for determining the exercise intensity remains unclear. As to the efficacy, a previous study ${ }^{6}$ have reported that PT was effective in improving ADLs in patients with MM. However, there are still few reports on the effectiveness of PT on MM patients, it is necessary to accumulate practical reports.

In this case report, we describe two cases in which patients with MM who presented with fractures or paralysis due to bone lesions, and whose general condition and ADLs were extremely reduced, improved through PT to ultimately receive autologous peripheral hematopoietic stem cell transplantation (auto-PBSCT) therapy. We conducted interventions focused on the management of exercise intensity using objective indicators, and considered the safety and effectiveness of our intervention from the process of two cases.

\section{PARTICIPANTS AND METHODS}

We present 2 patients with MM with Eastern Cooperative Oncology Group Performance Status (ECOG-PS) of 4 (i.e., they were completely disabled, unable to carry out self-care, and confined to the bed or chair) at the time of admission to our hospital. We fully explained the purpose of the case report to each patient verbally and obtained written consent.

The patients underwent resistance training for the upper and lower limbs in order to prevent disuse syndrome and ADL practice for 20 to 40 minutes twice a day for 6 days a week. The intensity of exercise was set at 13 (somewhat hard) on the Borg scale. The permissible range of bone pain was evaluated using the Common Terminology Criteria for Adverse Events v4.0 (CTCAEv4.0), an index for assessing adverse events in oncology ${ }^{7}$. The severity of bone pain is judged in three stages, from Grade 1 (mild pain) to Grade 3 (severe pain; limiting self-care ADL). Based on the reports of Cormie et al. ${ }^{3,4)}$ and Rief et al. ${ }^{5)}$, bone pain during exercise was limited to Grade 1 (mild pain) in both patients. If they experienced bone pain of Grade 2 (moderate pain; limiting instrumental ADL) or higher, or if the pain did not improve after resting, exercise was stopped and an orthopedist was consulted. As the role of each profession for pain management, the attending physician screened and followed up for osteolytic lesions using regular XP imaging, and the orthopedic surgeon performed regular follow-ups. The nurse managed to prevent falls in the patient's daily life during hospitalization and assisted in wearing a corset and ADL, according to the independence level. The physical therapist constantly exchanged information between attending the physician and nurse, and confirmed medical information and activities of daily living in the ward.

The decision on whether or not to perform PT during the chemotherapy myelosuppression period was based on the "criteria for discontinuing cancer rehabilitation ${ }^{8)}$ ". Specifically, when the leukocyte count was particularly low $(2,000 / \mu \mathrm{L}$ or less), the patients exercised in their ward or room to prevent infection. When the platelet count fell below $30,000 / \mu \mathrm{L}$, the patients underwent an "exercise program according to platelet count ${ }^{9)}$ ". If the hemoglobin level was less than $8 \mathrm{~g} / \mathrm{dL}$ and a blood transfusion was necessary, the patients exercised afterward. If the blood cell count fell below the standard value but the doctor judged that it was feasible to continue therapy, we intervened while considering the patient's physical condition. To determine the effect of PT, we measured ADLs using the Barthel index (BI) every 30 days from the start of PT. For Case 1 , the 6-minute walk distance (6MWD) was performed.

\section{RESULTS}

Case 1 was a 52 year-old male diagnosed with acute renal failure at a local hospital about 2.5 months before being transferred to the Department of Nephrology at a university hospital. He had a history of hypertension and diabetes. Computed tomography (CT) showed systemic bone lesions. He was referred to the Department of Hematology at the same hospital, where he was diagnosed with multiple myeloma (International Staging System stage 3 and Durie and Salmon stage 3 nonsecretory MM). He was treated with bortezomib and dexamethasone about 2 months before admission to our hospital but he did not respond. He was then treated with two courses of carfilzomib, lenalidomide, and dexamethasone (KRd), after which he was transferred to our hospital for continued treatment. He was able to do all ADLs without assistance before he became sick, but at the university hospital, he was bedridden because of the high risk of fractures due to osteolytic lesions throughout the body.

The functional status of Case 1 at the time of transfer to our hospital is as follows: at the time of admission to our hospital (Day 0), he was $165 \mathrm{~cm}$ tall and weighed $50.6 \mathrm{~kg}$; body mass index (BMI); $18.6 \mathrm{~kg} / \mathrm{m}^{2}$; and ECOG-PS 4. The X-ray photography (Xp) and magnetic resonance imaging (MRI) showed systemic osteolysis and compression fractures of the thoracic and lumbar spine (T4, T5, T6, T12, L1, and L2). The orthopedic surgeon allowed him to sit in an end sitting position with a soft corset for the thoracolumbar spine, and depending on the degree of pain, only get out of bed to do some movement, such as walking, while wearing a corset. On Day 2, he began the third course of KRd and PT. He could roll over independently, but needed moderate assistance to sit up and maximal assistance to stand up. It was difficult to maintain a sitting position for more than 5 minutes due to fatigue. He could maintain a standing position for 1 minute if both upper limbs were supported; however, both knee joints were hyperextended and there was a risk of sudden knee bending. The BI was 30 points (Fig. 1). For pain management, oxycodone extended release tablet ( $5 \mathrm{mg}$, twice a day) was prescribed from Day 0 to Day 6 and trans 


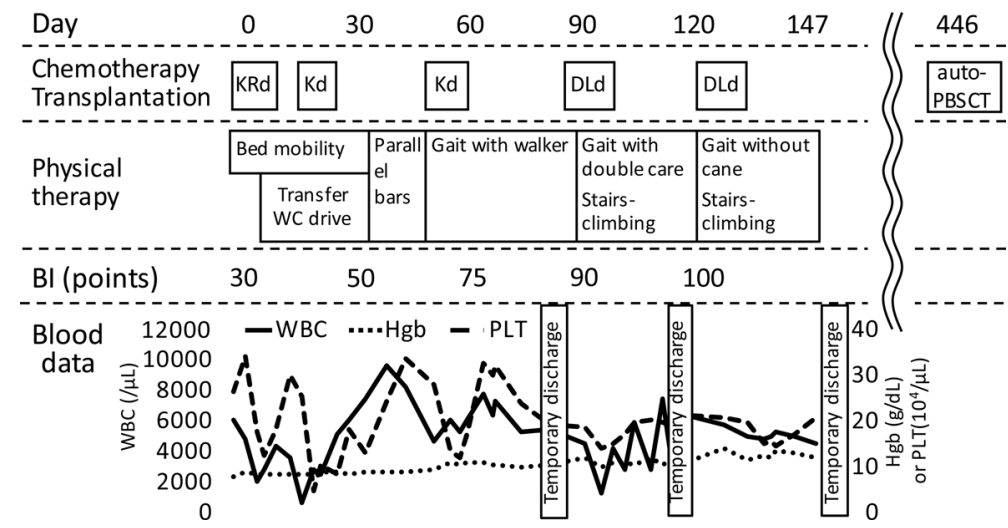

Fig. 1. Treatment and clinical course of Case 1.

KRd: carfilzomib + lenalidomide + dexamethasone; Kd: carfilzomib + dexamethasone; DLd: daratumumab + lenalidomide + dexamethasone; auto-PBSCT: autologous peripheral hematopoietic stem cell transplantation; WC: wheelchair; BI: Barthel index; WBC: leukocytes; Hgb: hemoglobin; PLT: platelets.

dermal fentanyl patch ( $1 \mathrm{mg}, 1$ sheet a day) was prescribed from Day 7 to Day 18. After Day 19, morphine oral solution $(5 \mathrm{mg}$ ) was administered according to the patient's pain. As for risk management in physical therapy, pain, swelling, and heat sensation were carefully confirmed during and after the physical therapy because pain sensitivity might be reduced due to drug therapy in addition to systemic osteolysis. In addition, a corset was always worn while leaving the bed to prevent excessive spinal flexion and rotation.

The progress to Day 30 is as follows: physical therapy centered on sit-to-stand and standing practice was started. He experienced mild bone pain (Grade 1 in CTCAEv4.0) in the lower back during exercise but no increase in pain after exercise. On Day 6, he developed fever, neutropenia, constipation, and vomiting, and KRd was discontinued. Chemotherapy was resumed on Day 16 with carfilzomib and dexamethasone $(\mathrm{Kd})$. According to the attending physician's instruction, the PT program from Day 6 to Day 13 was changed to exercise on the bed and practice maintaining a sitting position. From Day 14, sit-to-stand and standing practice was resumed, and transfer and wheelchair practice was added. On Day 27, he was able to stand up with minimal assistance, maintain a standing position for 2 minutes, self-propel in a wheelchair, and use a portable toilet. Pain during PT was mild in the lower back during movement (Grade 1 in CTCAEv4.0), and did not worsen. The BI improved to 50 points.

On Day 33, walking practice on parallel bars was started. However, on Day 51, he became nauseous while undergoing a second course of $\mathrm{Kd}$, and Thus $\mathrm{Kd}$ was discontinued until liver dysfunction improved. In physical therapy, exercise on the bed or discontinuation of PT continued for 5 days, but gradually resumed the standing up and walking practice. On Day 60, he could walk using a wheeled walker under close supervision, and the BI was 75 points. From Day 61, climbing up and down the steps practice and walking practice with two canes (Nordic walking poles) were performed. He could climb up and down the steps with the handrail, and could walk about $15 \mathrm{~m}$ with two canes. On Day 77, he was temporarily discharged home for 1 week. From Day 87, daratumab, lenalidomide, and dexamethasone (DLd) were started. In this period, he could safely walk alone using a wheeled walker, and became almost independent in ADLs (the BI was 90 points). There was no change in the degree of pain (Grade 1 in CTCAEv4.0).

After that, physical therapy was mainly performed to improve walking ability. In the 6 MWD using Nordic walking poles, it was $360 \mathrm{~m}$ on Day 103, but it extended to $450 \mathrm{~m}$ on Day 130. As of Day 120, BI was 100 points, and he could safely walk alone with two canes, and freehand walking was possible over $100 \mathrm{~m}$ under close supervision. On Day 147, he could safely walk alone freehand. His ECOG-PS was 1 (restricted in physically strenuous activity but ambulatory). The implementation rate of PT from Day 0 to Day 147 was as high as $98.3 \%$, and he was able to perform without adverse events.

Subsequently, chemotherapy was continued with repeated hospitalizations and discharges. In physical therapy, resistance training for the upper and lower limbs and bicycle ergometers were performed to improve his exercise tolerance. On Day 157, bone marrow examination confirmed the progression of MM, and DLd was discontinued. After he had received four courses of pomalidomide and dexamethasone by Day 258, he was then in partial remission and underwent peripheral blood stem cells harvest. On the Day 343, CT of the chest showed an increase in tumors of the left rib and hilum and pleural effusion. The attending physician determined that the disease had progressed and started him on ranimustine, vincristine, melphalan, and dexamethasone (ROAD) as salvage chemotherapy. On Day 387, CT of the chest showed that tumors of the left rib and hilum and pleural effusion had almost disappeared. The attending physician determined that chemotherapy was successful and he underwent a second course of ROAD. After Day 154, BI was maintained at 100 points, ECOG-PS was maintained between 1 and 2, and bone pain in the lower back was Grade 1 in CTCAEv4.0. On Day 446, he received auto-PBSCT.

Case 2 was a 53 year-old male who developed low back pain and weakness in both lower limbs about 3 months before 
admission of our hospital, and after that he was admitted to a local hospital for orthopedics because he had difficulty walking. He had a history of hypertension and diabetes. The MRI showed a compression fracture due to a T10 tumor, and he underwent posterior spinal fusion (T8-12) about 6 weeks later. Bone biopsy of the T10 vertebral arch showed a primary solitary plasmacytoma of the T10. He was treated with radiation therapy at a dose of $21 \mathrm{~Gy}$, but as the paralysis progressed, bortezomib, cyclophosphamide, and dexamethasone (VCD) was started about 10 days later. He developed bladder and rectal disorders associated with spinal cord injury, making urination difficult. A bladder catheter was placed, but he developed a urinary tract infection due to Pseudomonas aeruginosa. In addition, he developed deep vein thromboses in both lower limbs. He was able to perform all ADLs alone before he was sick, but at the local hospital he was mainly bedridden. Because he was single and living alone, and wanted treatment near where a relative lived, he was transferred to our hospital.

The functional status of Case 2 at the time of transfer to our hospital is as follows: at the time of admission, he was $175 \mathrm{~cm}$ tall and weighed $76.0 \mathrm{~kg}$; BMI, $24.8 \mathrm{~kg} / \mathrm{m}^{2}$; and ECOG-PS, 4. The X-rays showed that the bones had been fused, so ADLs were not limited if he wore a rigid corset (juet type) for the thoracolumbar spine. Figure 2 shows the course of treatment and PT at our hospital. On Day 1, the attending physician planned a total of four courses of VCD, and PT was started. The superficial and deep sensations of the patient's lower limbs were slightly dull and accompanied by spasticity. He could roll over alone but needed moderate assistance to sit up. He could maintain a sitting position with close supervision by using a bed fence, and transfer required maximal assistance. The pain was Grade 1 bone pain in CTCAEv4.0 in the lower back, but improved in the supine position. The BI was 20 points. As for pain management at our hospital, no drug was used in Case 2 , but attention was paid to pain during and after the physical therapy. In addition, a corset was always worn when leaving the bed to prevent excessive spinal flexion and rotation. PT planned resistance training in the lower limbs and practice sitting up, maintaining the end sitting position, and transferring.

The progress to Day 60 is as follows: on Day 10, wheelchair self-propelled practice was added to the PT program, which he could do alone. On Day 12, he could sitting up by using the back-raising function of the electric bed. On Day 24, transfer was possible with close supervision. In PT, maintaining a standing position practice, dressing practice, and recumbent ergometer were added. On Day 33, the orthopedist allowed exercise without a rigid corset. On Day 46, he was able to change clothes while still standing and to use the toilet with close supervision. On Day 59, walking practice was started, and he could walk about $10 \mathrm{~m}$ supported on parallel bars. Within this period, the pain had not changed significantly from that on Day 1 (Grade 1 in CTCAEv4.0). BI scored 35 points on Day 30 and 45 points on Day 60.

On Day 74, MRI showed obscuration of the tumor invading the spinal canal at T10 and relief of spinal cord compression. By this time, the pain in the lower back during and after exercise had almost disappeared. On Day 77, he could maintain a standing position for 3 minutes continuously and, to walk for $20 \mathrm{~m}$ or more continuously on parallel bars. From Day 89 , he started walking with a wheeled walker and could walk 50 m continuously. As of Day 90, the BI improved to 70 points. On Day 108, 4 courses of VCD were completed. He was able to urinate and the indwelling bladder catheter was removed. As of Day 120, BI was 80 points. From Day 136, he started walking practice with a 4-legged cane in his right hand and a single-legged cane in his left. He could walk $30 \mathrm{~m}$ continuously, but wobbling was observed, so moderate assistance was required. He could also walk $60 \mathrm{~m}$ continuously using a wheeled pickup walker with close supervision. On Day 145, the attending physician determined that his general condition and treatment status meant that outpatient treatment was possible. He was transferred from our hospital to a local hospital, where rehabilitation and management of plasmacytoma continued. During 145 days of staying at our hospital, the implementation rate of PT was 100\%, and the ECOG-PS was 3. Regarding Case 2, pain management by drug therapy was not required to be performed from the time of admission to our hospital, until the transfer to a local hospital.

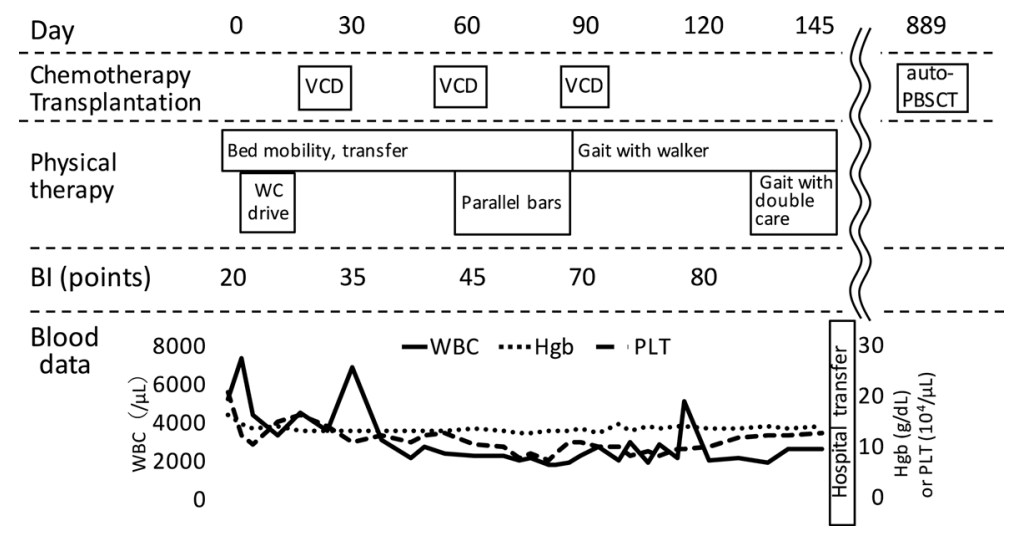

Fig. 2. Treatment and clinical course of Case 2.

VCD: bortezomib + cyclophosphamide + dexamethasone; auto-PBSCT: autologous peripheral hematopoietic stem cell transplantation; WC: wheelchair; BI: Barthel index; WBC: leukocytes; Hgb: hemoglobin; PLT: platelets. 
After that, he continued PT at the local hospital and was able to walk safely alone with a forearm crutch in one hand. The ECOG-PS improved to 1. On Day 669, plasmacytoma progressed to MM, so four courses of KRd were performed from Day 670 to Day 752, resulting in partial remission. On Day 889, he received auto-PBSCT.

\section{DISCUSSION}

Although it is necessary for patients with MM to exercise at an appropriate intensity for effectively improving ADLs, risk management of the bone lesions is also very important. Exercise intensity of 13 on the Borg scale is widely used in cancer patients after surgery and during chemotherapy ${ }^{10-13)}$ and reported to be a safe exercise intensity in malignant lymphoma, which is a hematopoietic malignancy ${ }^{14}$. However, it was not clear whether the intensity of 13 on the Borg scale is safe in patients with MM and patients with reduced ECOG-PS.

Therefore, we retrospectively examined the safety and effectiveness of PT wherein exercise intensity was set at 13 on the Borg scale and Grade 1 in the CTCAEv4.0. As a result of our intervention on two cases of MM who presented with fractures or paralysis due to bone lesions, no adverse events occurred despite long-term intervention. Additionally, the implementation rate of PT was very high in both patients. This result suggests that interventions based on the Borg scale and CTCAEv4.0 are highly safe for patients with MM. Furthermore, ADLs and ECOG-PS were improved in both patients. This suggests that the exercise intensity was also appropriate in terms of effectiveness and was not too weak. Therefore, the present case report demonstrates that exercise intensity and risk management using 13 on the Borg scale and Grade 1 in the CTCAEv4.0 can help perform safe and effective PT in patients with MM.

Both patients received auto-PBSCT therapy after chemotherapy and PT. Auto-PBSCT is regarded as the optimal treatment for patients with MM having good ECOG-PS scores ${ }^{15)}$. Child et al. ${ }^{16)}$ reported that among patients with MM who underwent auto-PBSCT ( $\mathrm{n}=401)$, ECOG-PS corresponded to 0 in $15 \%$ and 1 in $59 \%$. This suggests that a large proportion of patients with MM who receive auto-PBSCT have PS 0 or 1. Therefore, Improving ADLs in patients with MM with poor ECOG-PS scores may broaden the range of treatment methods. In the patients presented in the current study, the ECOG-PS was 4 at the time of admission, but had improved to 1 or 2 before auto-PBSCT was initiated. In other words, the improvement in ADLs by PT may have contributed to performing the auto-PBSCT in two patients with MM.

One limitation in the study is that only two patients were studied, making it difficult to state the appropriate setting of exercise intensity and contribution to auto-PBSCT by PT based on this result alone. In addition, the effect of PT on health-related quality of life was not examined in this study. A recent previous study reported that there was no change in health-related quality of life before and after the 12-week multidisciplinary rehabilitation program in MM patients ${ }^{17}$. In the future, it is necessary to study more such cases and verify the safety and effectiveness of PT including health-related quality of life in patients with MM.

\section{Conflict of interest}

The authors declare no conflicts of interest with any individuals or companies.

\section{REFERENCES}

1) Japanese Society of Myeloma: Guideline for MM, 4th ed (in Japanese). Tokyo: Bunkodo, 2016, p 140.

2) Matsumoto M, Tuji T: Rehabilitation of pain associated with bone metastasis. Pain Clin, 2012, 33: 1391-1401.

3) Cormie P, Galvão DA, Spry N, et al.: Functional benefits are sustained after a program of supervised resistance exercise in cancer patients with bone metastases: longitudinal results of a pilot study. Support Care Cancer, 2014, 22: 1537-1548. [Medline] [CrossRef]

4) Cormie P, Newton RU, Spry N, et al.: Safety and efficacy of resistance exercise in prostate cancer patients with bone metastases. Prostate Cancer Prostatic Dis, 2013, 16: 328-335. [Medline] [CrossRef]

5) Rief H, Welzel T, Omlor G, et al.: Pain response of resistance training of the paravertebral musculature under radiotherapy in patients with spinal bone metastases--a randomized trial. BMC Cancer, 2014, 14: 485. [Medline] [CrossRef]

6) Kamiya T, Morisaka A, Iwasaki T, et al.: Clinical study of the physical therapy for the Multiple Myeloma patients in our hospital. J Aichi Soc Phys Ther, 2015, 27: $60-64$.

7) US Department of Health \& Human Services. Common Terminology Criteria for Adverse Events (CTCAE). [Updated June 14, 2010] https://evs.nci.nih.gov/ ftp1/CTCAE/CTCAE_4.03/CTCAE_4.03_2010-06-14_QuickReference_5x7.pdf. (Accessed May 14, 2016)

8) Tsuji T, Kimura A: Oncological rehabilitation-overview. Sogo Rihabiriteshon, 2003, 31: 753-760 (in Japnaese).

9) Tsuji T: Best practices for cancer rehabilitation. Japan Cancer Rehabilitation Study Group (ed.). Tokyo: Kanehara \& Co., 2015 , pp 10-26.

10) Hacker ED, Larson JL, Peace D: Exercise in patients receiving hematopoietic stem cell transplantation: lessons learned and results from a feasibility study. Oncol Nurs Forum, 2011, 38: 216-223. [Medline] [CrossRef]

11) Dimeo FC, Thomas F, Raabe-Menssen C, et al.: Effect of aerobic exercise and relaxation training on fatigue and physical performance of cancer patients after surgery. A randomised controlled trial. Support Care Cancer, 2004, 12: 774-779. [Medline] [CrossRef]

12) Thorsen L, Skovlund E, Strømme SB, et al.: Effectiveness of physical activity on cardiorespiratory fitness and health-related quality of life in young and middle-aged cancer patients shortly after chemotherapy. J Clin Oncol, 2005, 23: 2378-2388. [Medline] [CrossRef]

13) van Waart H, Stuiver MM, van Harten WH, et al.: Effect of low-intensity physical activity and moderate- to high-intensity physical exercise during adju- 
vant chemotherapy on physical fitness, fatigue, and chemotherapy completion rates: results of the PACES randomized clinical trial. J Clin Oncol, 2015, 33: 1918-1927. [Medline] [CrossRef]

14) Kasahara R, Fujita T, Takahashi S, et al.: Feasibility of an exercise therapy in lymphoma patients with cytopenia: preliminary study by retrospective observational study. J Jpn Phys Ther Assoc, 2020, 47: 174-180.

15) Fermand JP, Katsahian S, Divine M, et al. Group Myelome-Autogreffe: High-dose therapy and autologous blood stem-cell transplantation compared with conventional treatment in myeloma patients aged 55 to 65 years: long-term results of a randomized control trial from the Group Myelome-Autogreffe. J Clin Oncol, 2005, 23: 9227-9233. [Medline] [CrossRef]

16) Child JA, Morgan GJ, Davies FE, et al. Medical Research Council Adult Leukaemia Working Party: High-dose chemotherapy with hematopoietic stem-cell rescue for multiple myeloma. N Engl J Med, 2003, 348: 1875-1883. [Medline] [CrossRef]

17) Nielsen LK, Larsen RF, Jarlbaek L, et al.: Health-related quality of life in patients with multiple myeloma participating in a multidisciplinary rehabilitation program. Ann Hematol, 2021. [Medline] [CrossRef] 